\title{
Old and New Prognostic Markers in Pediatric ALL
}

\author{
Hara Prasad Pati ${ }^{1} \cdot$ Prashant Sharma $^{1}$
}

Published online: 23 February 2016

(C) Indian Society of Haematology \& Transfusion Medicine 2016

Dear Readers,

Welcome to the spring issue of the IJHB, with a fresh bouquet of articles for you.

In this issue, Aydin et al. [1] compare conventional cytogenetics with FISH and RT-PCR testing for $\mathrm{t}(12 ; 21)(\mathrm{p} 13 ; \mathrm{q} 22)$ in pediatric B-acute lymphoblastic leukemia (ALL) patients. This disease represents an outstanding example, together with APML and CML, of modern medicine's triumph over hematological malignancies using a combination of monitoring and risk-adapted therapeutic strategies [2]. The development and evolution of minimal residual disease (MRD) analysis by the relatively widely available flow cytometric techniques has rapidly made MRD the most decisive prognostic marker in ALL [3]. Standardization and availability remain pertinent issues in India, but the rate of dissemination of knowledge of the technique and its application remain enthralling examples of how quickly research is translating into better patient management.

On the molecular front in B-ALL, the importance of $b c r$-abll fusion, $M L L$ rearrangements, DNA ploidy, $\mathrm{t}(12 ; 21)$ and other prognostic markers is well-documented. Their occurrence in $80-90 \%$ patients makes them widely applicable as well [2]. More recently, genome-wide analyses and high throughput sequencing have uncovered multiple key pathways frequently mutated in B-ALL. New ALL subtypes, most prominently, Philadelphia $(\mathrm{Ph})$ chromosome-like ALL have been recognized based on abnormalities of lymphoid development, tumour suppression,

\footnotetext{
$\triangle$ Hara Prasad Pati

harappati@yahoo.co.in

1 New Delhi, India
}

chromatin remodelling, intracellular kinases and cytokine receptors, and their downstream interactions with their ligands/regulators. High-risk Ph-like ALL is especially important since it is characterized by tyrosine kinase activation that can be inhibited with currently available pharmacological agents [4, 5]. Genomic profiling has also aided identification of inherited risk factors for leukaemia development including GATA3, IKZF1, CEBPE and CKDN2A [6].

FISH or PCR testing reveals the $\mathrm{t}(12 ; 21)$ in $\sim 25 \%$ pediatric B-ALL, compared to only $2-3 \%$ adults. The translocation is cryptic in the vast majority of cases, being undetectable by conventional karyotyping [7]. The resultant ETV6-RUNX1 (previously called the TEL$A M L 1)$ fusion protein converts RUNX1 from a transcriptional activator to a repressor. Since $R U N X I$ is normally required for the expression of several hematopoiesisspecific genes as well as for lineage-specific transcription, its impact on leukemogenesis is profound. Clinically, ETV6-RUNX1 is a good prognostic factor, found in children aged 1-10 years, with CD10 + ALL and characterized by late relapses with excellent chemosensitivity $[2$, 7]. From a laboratory perspective, as the Aydin paper [1] illustrates, FISH probes for the ETV6/RUNX1 fusion also enable presumptive identification of aneuploidies of chromosomes 12 or 21 as well as ETV6or RUNX1 deletions or amplifications. The poor prognostic intra-chromosomal amplification of chromosome 21 (iAMP21) is also detectable by this modality $[7,8]$.

In conclusion, the march continues towards better and more insightful prognostic tests for hematological malignancies. Upgrading our national laboratories to ensure that all patients with B-ALL receive timely, accurate and costeffective testing should be a priority with the hematology community. 


\section{References}

1. Aydin C, Cetin Z, Manguoglu AE et al. Evaluation of ETV6/ RUNX1 fusion and additional abnormalities involving ETV6 and/ or RUNX1 genes using FISH technique in patients with childhood acute lymphoblastic leukemia. Indian J Hematol Blood Transfus $1-8$

2. Prakash G, Kaur A, Malhotra P et al (2016) Current role of genetics in hematologic malignancies. Indian $\mathrm{J}$ Hematol Blood Transfus 32:18-31

3. Ravandi F, Jorgensen JL, O'Brien SM et al (2016) Minimal residual disease assessed by multi-parameter flow cytometry is highly prognostic in adult patients with acute lymphoblastic leukaemia. Br J Haematol 172(3):392-400

4. Roberts KG, Li Y, Payne-Turner D et al (2014) Targetable kinaseactivating lesions in Ph-like acute lymphoblastic leukemia. N Engl J Med 371:1005
5. Roberts KG, Mullighan CG (2015) Genomics in acute lymphoblastic leukaemia: insights and treatment implications. Nat Rev Clin Oncol 12(6):344-357

6. Moriyama T, Relling MV, Yang JJ (2015) Inherited genetic variation in childhood acute lymphoblastic leukemia. Blood 125:3988-3995

7. Moorman AV, Ensor HM, Richards SM et al (2010) Prognostic effect of chromosomal abnormalities in childhood B-cell precursor acute lymphoblastic leukaemia: results from the UK Medical Research Council ALL97/99 randomised trial. Lancet Oncol $11: 429$

8. Strefford JC, van Delft FW, Robinson HM et al (2006) Complex genomic alterations and gene expression in acute lymphoblastic leukemia with intrachromosomal amplification of chromosome 21. Proc Natl Acad Sci USA 103:8167-8172 